\begin{tabular}{|c|c|}
\hline Citation & $\begin{array}{l}\text { Frank P. Luyten, Matteo Denti, Giuseppe Filardo, Elizaveta Kon, Lars } \\
\text { Engebretsen, (2012), } \\
\text { Definition and classification of early osteoarthritis of the knee, } \\
\text { Knee Surgery, Sports Traumatology, Arthroscopy, 20(3), 401-406. }\end{array}$ \\
\hline Archived version & $\begin{array}{l}\text { Author manuscript: the content is identical to the content of the published } \\
\text { paper, but without the final typesetting by the publisher }\end{array}$ \\
\hline Published version & http://dx.doi.org/10.1007/s00167-011-1743-2 \\
\hline Journal homepage & http://www.springer.com/medicine/orthopedics/journal/167 \\
\hline Author contact & $\begin{array}{l}\text { frank.luyten@uz.kuleuven.ac.be } \\
+32(0) 16342541\end{array}$ \\
\hline IR & https://lirias.kuleuven.be/handle/123456789/340351 \\
\hline
\end{tabular}

(article begins on next page) 



\title{
KULEUVEN
}

\section{Definition and classification of early osteoarthritis of the knee}

\author{
Frank P. Luyten ${ }^{a}$, Matteo Denti $^{b}$, Giuseppe Filardo $^{c}$, Elizaveta Kon $^{c}$, Lars Engebretsen $^{d, e}$
}

${ }^{a}$ Division of Rheumatology, Laboratory for Skeletal Development and Joint Disorders, K.U. Leuven, Herestraat 49, Bus 813, 3000 Leuven, Belgium

${ }^{\mathrm{b}}$ Knee Surgery and Sports Traumatology,IRCCS Istituto Clinico Humanitas,

Via Manzoni 56, Rozzano, Milan, Italy

${ }^{c}$ Biomechanics Laboratory, III Clinic, Rizzoli Orthopaedic Institute, Via di Barbiano 1/10, 40136 Bologna, Italy

${ }^{\mathrm{d}}$ Department of Sports medicine, Oslo Sports Trauma Research Center,

Norwegian School of Sport Sciences, 608 Oslo, Norway

${ }^{\mathrm{e}}$ Department of Orthopaedic Surgery, University of Oslo, Oslo, Norway 


\section{KU LEUVEN}

\section{Abstract}

With the emerging interest in regenerative medicine and tissue engineering, new treatment modalities are being developed for joint disorders including joint surface lesions and articular cartilage defects. The clinical outcome of these novel approaches appears rather unpredictable, and is due to many reasons but definitely also linked to the patient profile. As a typical example, symptomatic articular cartilage lesions can be presented in an otherwise normal joint, or associated with several other joint tissue alterations including meniscal lesions and abnormalities of the underlying bone. The outcome of novel treatments may well be influenced by the status of the whole joint, and the potential to develop osteoarthritis. To better identify the patients at risk and responders to certain treatments, it is of use to define and most importantly classify patients with "early osteoarthritis". Here classification criteria for this group of patients are presented, allowing a more defined and accurate inclusion in clinical trials in the future.

\section{Key words}

early osteoarthritis, classification, knee, MRI, arthroscopy 


\section{Introduction}

An increasing number of clinical studies in the field of joint surface repair have revealed that several treatment approaches may be successful in improving clinical outcome on the short and long term [19]. It becomes clear after a glance on recent literature research that patient populations with symptomatic cartilage defects are heterogeneous, some having isolated joint surface defects in otherwise perfectly healthy joints, others, however, showing associated joint lesions frequently resulting in loss of joint homeostasis. In order to better appreciate and understand outcomes from clinical studies and new treatments, it appears relevant to improve the characterization of the patient populations. Indeed, it is observed in trials and clinical practice that outcomes may be quite different depending on the patient profile. Identifying patients at risk for progression seems imperative to better assess treatment response. Therefore, in addition to the classification criteria of established osteoarthritis (OA) of the knee $[1,11]$, classification criteria for an early OA population have been proposed, aiming to identify a subpopulation of patients with signs of emerging joint disease and probably at a higher risk to develop frank OA of the knee. With the increasing availability of novel biological treatments such as Platelet Rich Plasma (PRP) [9] and other more regenerative treatment approaches, including the new class of Advanced Therapeutic Medicinal Products [16], it is useful to better characterize the patient populations entering these clinical studies, thereby contributing to the major goals of modern, more personalized medicine approaches i.e. identifying the patients at risk and responders to treatment.

Importantly, classification criteria do not serve to establish a diagnosis in individual patients, but are merely used to allow to compare on a large, worldwide scale, patient groups/populations with specific comparable characteristics, typically for clinical trial purposes.

\section{Defining and diagnosis of early knee OA}

The diagnosis of knee OA can usually be made by history and physical examination including signs/symptoms of knee pain with stiffness, joint crepitus and functional limitations, typically in a population above 50 years old. Diagnosis is confirmed by radiographs demonstrating changes such as osteophytes and joint space narrowing, subchondral bone sclerosis and cysts, and graded according to Kellgren and Lawrence as Kellgren II-IV [6,13]. Early OA of the knee is somewhat more complicated as the signs/symptoms may still be limited and sporadic, only becoming manifest under certain conditions such as after long term loading (jogging or other sports activities). It is thought to be a process that displays a number of tissue related phenomena leading to the loss of homeostasis of the knee, and in most cases leading to established OA. It is the clinical recurrence of pain and discomfort of the knee, short periods of stiffness, with in between long periods of very little clinical manifestations, probably due to spontaneous adaption of the patient, that sets the stage to perform additional investigations such as radiographs, ultrasound, MRI or arthroscopy. Frequently, in these cases, the history and the clinical examination suggest a local problem of mechanical nature with no systemic manifestations. Classical radiographs in general are quite disappointing in this sense that, certainly if no earlier radiographs are available as in most cases, very few specific signals are seen, at best there is a suggestion on some joint space narrowing in one compartment, some hints for the formation of what may probably become an osteophyte, and thus typically at best qualified by a Kellgren I. The robustness of this last scoring category is however difficult, and studies reveal the quite poor intra- and interreader reproducibility of this scoring system $[11,13]$. In addition, it reveals very little of potentially many more tissue processes in the joint. New imaging techniques rapidly improving in quality over the last years, in particular MRI and arthroscopy (which is seldom used as a pure 
diagnostic tool), have revealed a spectrum of joint tissue changes that definitely identify more pathologies in the joint, and loss of joint homeostasis. These tissue changes typically display, in one of more compartments of the knee, changes in cartilage morphology with joint surface fibrillation and single or multiple cartilage defects, more diffuse cartilage loss, meniscal damage with tears, degeneration and extrusion of the meniscus, bone marrow lesions (BMLs), subchondral sclerosis and cysts, synovitis and presence of joint fluid to mention the most important ones. In these cases, arthroscopy and MRI are to a certain extent complementary thereby providing an overall assessment of the knee and its structural changes. It is precisely with these tools available in daily clinical practice, that it is clear that in a number of patients it should be possible to define an early OA patient. Early OA would typically combine clinical signs and symptoms as described above, but also displaying a number of structural changes that are not captured by regular radiographs, in many cases having a protocol of normal findings, and yet revealing by in depth imaging more changes identifying a knee potentially in danger to progress and develop frank OA. In lieu of the development of these technologies, it is surprising that the OA definition has not changed since 1986 to capture OA, and certainly early OA. In view of an increasing number of clinical investigations and trials with innovative treatment approaches, it appears to be useful to classify early OA, and discriminate this from established OA patients and patients with structural damage, but further healthy joints. Indeed, sub analyses of patient populations in clinical practice suggest a potentially different outcome for treatment approaches and this requires thus a more targeted approach for future studies.

\section{Classification criteria for early knee OA}

The American College of Rheumatology (ACR) criteria for OA of the knee have been published by Altman et al. [1]. These classification criteria, used typically as inclusion criteria in clinical trials worldwide, are mostly the combination of clinical and radiographic findings. They include one of the following three findings, age above 50, stiffness less than $30 \mathrm{~min}$, and crepitus, together with structural changes i.e. osteophytes and joint space narrowing (Kellgren II on standardized radiographs). These classification criteria display a $91 \%$ sensitivity and $86 \%$ specificity. Recently, in an attempt to harmonize studies addressing the underlying genetic basis of $\mathrm{OA}$, Kerkhof $\mathrm{HJ}$ et al. [7] published recommendations on standardization of OA phenotypes, suggesting that at least one definite osteophyte with possible joints space narrowing is needed to establish the diagnosis of radiological knee OA. Additional issues around the OA knee phenotype have been reported in other publications, highlighting the pitfalls in defining symptomatic and radiological OA [4].

Defining classification criteria of symptomatic early knee OA is certainly challenging, but is obviously based on the fact that the patient cannot be classified as established OA. To make it clinically relevant, and to help classifying patients for clinical trials, would still imply the combination of symptoms, signs, and structural changes. However, strict radiographic criteria as defined by Kellgren will not suffice to capture an early OA population. Therefore, a more comprehensive classification allowing other methods of structural assessment such as arthroscopy and MRI are proposed.

As suggested above, and in view of the existing classification criteria for OA, the following criteria are proposed. A patient can be classified as having early OA of the knee based on clinical and imaging findings and should fulfill the following three criteria (Tab. 1):

1. Pain in the knee.

2. Standard radiographs: Kellgren-Lawrence grade 0 or I or II (osteophytes only).

3. At least one of the two following structural criteria: 
- $\quad$ Arthroscopic findings of cartilage lesions.

- MRI findings demonstrating articular cartilage degeneration and/or meniscal degeneration, and/or subchondral BMLs.

More detailed description of classification criteria

1. Knee pain: at least two episodes of pain for $>10$ days in the last year.

2. Standard radiographs: Kellgren-Lawrence scoring up to II (osteophytes only) in standing weight-bearing position with knees in approximately $20^{\circ}$ of flexion and the feet in $5^{\circ}$ of external rotation. The radiographs should be done bilaterally from a posteroanterior view in the frontal plane. Kellgren-Lawrence grade 0 is no abnormalities. Kellgren-Lawrence I is defined as: doubtful narrowing of the joint space and possible osteophytic lipping. Kellgren-Lawrence II is defined as definite osteophytes with joint space narrowing. In agreement with a recent adjustment [4], Kellgren II/ osteophytes (osteophytes only, no joint narrowing) has been introduced into the category early OA (Tab. 2).

3. Arthroscopic findings following the ICRS classification [20]:

ICRS grade I-IV in at least two compartments or grade II-IV in one compartment with at least surrounding softening and swelling of the cartilage.

4. MRI findings: evidence of degenerative changes of the cartilage, meniscus and/or BMLs. The definitions are based on the BLOKS and WORMS scores $[5,12]$ and their comparisons $[3,10]$. Minimum two of the four following scores should be fulfilled:

a. Cartilage morphology scores grade 3 or higher (WORMS grade 3 to 6 ): minimally multiple areas of partial thickness defects with intermittent areas of normal thickness to diffuse full thickness loss in region (more then 75 \%) (grade 6) (Fig. 1).

b. Cartilage Score 1: minimally grade 2 (BLOKS grade 2 and 3): 10-75\% of cartilage loss in a region (medial, lateral, patellofemoral) to more than $75 \%$ cartilage loss in a region (Tab. 3).

c. Meniscal tears: Grade 3 or higher (BLOKS grade 3-4): from displaced tears or partial resection (grade 3) to complete maceration, destruction, resection (grade 4) (Tab. 4).

d. BMLs, typically scored as BMLs size: minimally WORMS grade 2 i.e. $25 \%$ or higher BMLs in any one compartment (Fig. 2).

\section{Discussion}

In this paper criteria to classify patients with "early OA" have been defined.

New treatment approaches in the so called field of regenerative medicine (REGMED) have been emerging in the field of musculoskeletal diseases and disorders. These treatments are based on enhancing the intrinsic repair system, and typically involve surgical interventions or the application of growth and differentiation factors or other biological substances, sometimes combined with surgical delivery systems. Amid these REGMED developments, a new class of products, also called Advanced Therapeutic Medicinal Products, has been defined with a new regulatory track as proposed by EMA in 2007 and amended in 2009 [17-18]. Among these are also the cell based products, ranging from cellular suspensions with or without delivery vehicles, combination products, typically scaffold based living implants, and more advanced tissue engineered products. Many of these novel treatments have shown great promise in various musculoskeletal indications, but the results in the limited number of well designed clinical studies are revealing a number of challenges, including the value of subjective and objective outcomes, their discriminatory power and functional assessments in the short and long term. In view of this, it has been 
observed in clinical practice, but also in open and controlled clinical studies, that treatment approaches in the knee for cartilage lesions or some other indications are resulting in the recruitment of a patient population that is quite heterogeneous. For instance, the Knutsen et al. trial [8], comparing different treatment methods for the repair of symptomatic cartilage lesions in the knee, reported after 5 years up to $35 \%$ of patients displaying frank OA as assessed by radiography. This is in sharp contrast to the Saris, van Lauwe et al. trial reports even after 5 years less than $5 \%$ of the patients assessed displayed definite osteophytes [14,15] (Luyten F, personal communication). This indicates indeed that although the inclusion criteria of both trials were quite comparable, the patient populations were most probably not the same, and thus outcomes may be influenced by the patient profile. On the other hand, it is very difficult to use strict inclusion criteria when recruiting patients with cartilage problems to a controlled study. As shown recently, cartilage defect patients represent a mixed group in terms of age, size of defect, anatomical location of defect, co-injuries and previous surgery [2]. A reader of an RCT that does not present a flowchart of the patient selection runs the risk of misjudging the results when interpreting the paper. Additionally, the variations found in inclusion criteria in the published RCTs represent a concern related to whether the studies actually include the same patient groups. This is certainly not restricted to these trials, but is also a challenge in other fields of medicine as shown by the applicability of RCTs to the general patient population. Defining early OA may help to distinguish and understand outcomes more clearly. Therefore, the current paper presents a classification of early OA based on symptoms (pain), structural changes as defined by regular radiographs (correct projections are of primary importance) by Kellgren Lawrence of II (osteophytes only) or less, and early degenerative changes as detected by either MRI scores or arthroscopic findings. Indeed, for the first time arthroscopic exam and/or MRI findings are now taken into account, but always combined with symptoms. This is a dynamic definition and may in the future be modified by new data coming from new technological developments such as dGEMRIC-MRI and other MRI systems, Ultrasound and/or Biomarkers.

The criteria put forward for early degenerative changes in arthroscopy are limited to the scoring of the articular surface, and do not include meniscal evaluations. Indeed, scoring menisci by arthroscopy is difficult and always incomplete.

MRI is very useful to score the full thickness articular cartilage, and since the WORMS and BLOKS score are only partially overlapping, the WORMS rather scoring overall cartilage morphology, both scoring systems for the cartilage have been kept. Concerns may rise on the inclusion of the meniscal assessment, as meniscal lesions may solely be the result of an isolated traumatic event and not reflecting early OA. That is an important reason to rely on at least two criteria to be able to classify MRI findings as early OA, meniscal damage in itself is thus never sufficient and needs to be accompanied by either substantial joint cartilage involvement and/or BMLs.

MRI, and in particular the WORMS score, appears to be reliable for BMLs but is not good to assess subchondral bone sclerosis. However, scoring of cysts would not be helpful to identify early OA. Also, as published in Lynch et al. [10], scores do not identify a lot a primarily cystic BMLs in an OA population and scoring these did not alter much in the final scoring of the patient.

The definition of clear classification criteria will help to better design trials for the assessment of the potential and indications of the treatments of early OA, and therefore to better apply the available treatment options and to better manage patients affected by cartilage degeneration in the clinical practice. 


\section{Conclusions}

In view of the emerging new treatment approaches, it may be beneficial to clearly distinguish patient populations with knee pathology, i.e. patients with tissue lesions (meniscus, cartilage, ligaments) but otherwise healthy joints, patients with lesions in the context of early OA, displaying a number of changes in the joint tissues suggestive of emerging joint disease, and finally well established $O A$ as defined by the existing ACR criteria. That implies that clinical trial designs should be taking this into account, and helpto identify patients at risk and responders to treatment. This will hopefully lead to improved treatment algorithms in specific patient populations. The above presented classification criteria appear very practical and applicable in daily practice, but as new insights and technologies become available they may and will most probably further evolve. 


\section{REFERENCES}

[1] Altman R, Asch E, Bloch D, Bole G, Borenstein D, Brandt K et al (1986) Development of criteria for the classification and reporting of osteoarthritis. Classification of osteoarthritis of the knee. Diagnostic and Therapeutic Criteria Committee of the American Rheumatism Association. Arthritis Rheum 8:1039-1049

[2] Engen CN, Engebretsen L, Aroen A (2010) Knee cartilage defect patients enrolled in randomized controlled trials are not representative of patients in orthopedic practice. Cartilage 312-319

[3] Felson DT, Lynch J, Guermazi A, Roemer FW, Niu J, McAlindon T et al (2010) Comparison of BLOKS and WORMS scoring systems part II. Longitudinal assessment of knee MRIs for osteoarthritis and suggested approach based on their performance: data from the Osteoarthritis Initiative. Osteoarthritis Cartilage 11:1402-1407

[4] Felson DT, Niu J, Guermazi A, Sack B, Aliabadi P (2011) Defining radiographic incidence and progression of knee osteoarthritis: Problems with the Kellgren and Lawrence scale. Ann Rheum Dis 2011 70:1884-1886

[5] Hunter DJ, Lo GH, Gale D, Grainger AJ, Guermazi A, Conaghan PG (2008) The reliability of a new scoring system for knee osteoarthritis $\mathrm{MRI}$ and the validity of bone marrow lesion assessment: BLOKS (Boston Leeds Osteoarthritis Knee Score). Ann Rheum Dis 2:206-211

[6] Kellgren JH, Lawrence JS (1957) Radiological Assessment of Osteo-Arthrosis. Annals of the Rheumatic Diseases 4:494-502

[7] Kerkhof HJ, Meulenbelt I, Akune T, Arden NK, Aromaa A, Bierma-Zeinstra SM et al (2011) Recommendations for standardization and phenotype definitions in genetic studies of osteoarthritis: the TREAT-OA consortium. Osteoarthritis Cartilage 3:254-264

[8] Knutsen G, Drogset JO, Engebretsen L, Grontvedt T, Isaksen V, Ludvigsen TC et al (2007) A randomized trial comparing autologous chondrocyte implantation with microfracture. Findings at five years. J Bone Joint Surg Am 10:2105-2112

[9] Kon E, Buda R, Filardo G, Di Martino A, Timoncini A, Cenacchi A et al (2010) Platelet-rich plasma: intra-articular knee injections produced favorable results on degenerative cartilage lesions. Knee Surg Sports Traumatol Arthrosc 4:472-479

[10] Lynch JA, Roemer FW, Nevitt MC, Felson DT, Niu J, Eaton CB et al (2010) Comparison of BLOKS and WORMS scoring systems part I. Cross sectional comparison of methods to assess cartilage morphology, meniscal damage and bone marrow lesions on knee MRI: data from the osteoarthritis initiative. Osteoarthritis Cartilage 11:1393-1401

[11] Oiestad BE, Holm I, Aune AK, Gunderson R, Myklebust G, Engebretsen L et al (2010) Knee function and prevalence of knee osteoarthritis after anterior cruciate ligament reconstruction: a prospective study with 10 to 15 years of follow-up. Am J Sports Med 11:2201-2210

[12] Peterfy CG, Guermazi A, Zaim S, Tirman PF, Miaux Y, White D et al (2004) Whole-Organ Magnetic Resonance Imaging Score (WORMS) of the knee in osteoarthritis. Osteoarthritis Cartilage 3:177-190

[13] Risberg MA, Holm I, Tjomsland O, Ljunggren E, Ekeland A (1999) Prospective study of changes in impairments and disabilities after anterior cruciate ligament reconstruction. J Orthop Sports Phys Ther 7:400-412

[14] Saris DB, Vanlauwe J, Victor J, Almqvist KF, Verdonk R, Bellemans J et al (2009) Treatment of symptomatic cartilage defects of the knee: characterized chondrocyte implantation results in better clinical outcome at 36 months in a randomized trial compared to microfracture. Am J Sports Med 9;10S-9S

[15] Saris DB, Vanlauwe J, Victor J, Haspl M, Bohnsack M, Fortems $Y$ et al (2008) Characterized chondrocyte implantation results in better structural repair when treating symptomatic cartilage defects of the knee in a randomized controlled trial versus microfracture. Am J Sports Med 2:235-246

[16] Schneider CK, Salmikangas P, Jilma B, Flamion B, Todorova LR, Paphitou A et al (2010) Challenges with advanced therapy medicinal products and how to meet them. Nat Rev Drug Discov 3:195-201

[17] The European Parliament and the Council of the European Union (2007) Regulation (EC) No. 1394/2007 of the European Parliament and of the Council of 13 November 2007 on advances therapy medicinal products and amending directive 2001/83/EC and regulation (EC) No 726/2004. Official J Eur Union

[18] The European Parliament and the Council of the European Union (2009) Commission Directive 2009/120/EC of 14 September 2009 amending Directive 2001/83/EC of the European Parliament and of the Council on the Community code relating to medicinal products for human use as regards advanced therapy medicinal products. Official J Eur Union

[19] Van Osch GJ, Brittberg M, Dennis JE, Bastiaansen-Jenniskens YM, Erben RG, Konttinen YT et al (2009) Cartilage repair: past and future--lessons for regenerative medicine. J Cell Mol Med 5:792-810

[20] www.cartilage.org/_files/contentmanagement/ICRS_evaluation.pdf 


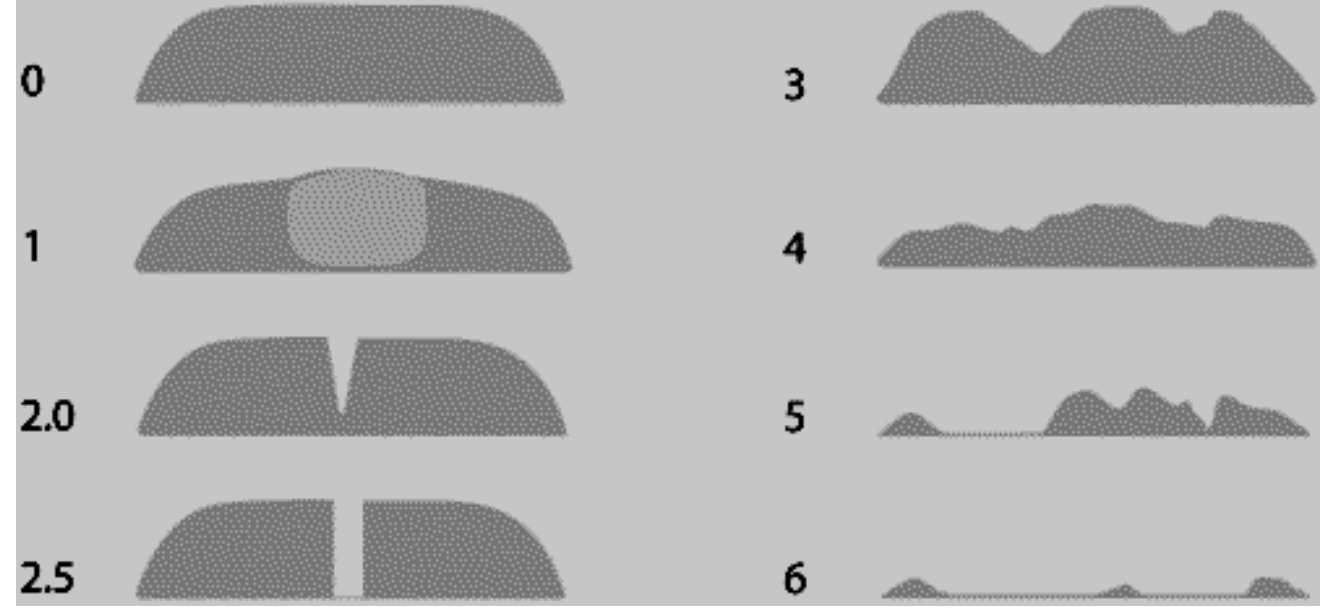

Figure 1: Cartilage morphology scores (WORMS grading) [12]

Eight-point scale for scoring articular cartilage signal and morphology.

Each region of the knee surface is scored independently.

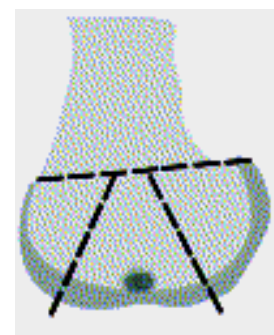

1

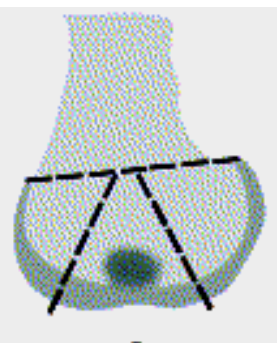

2

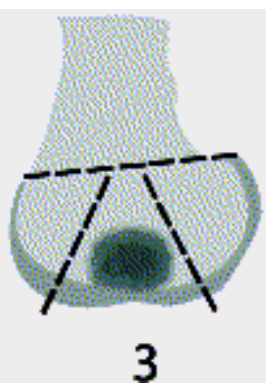

3

Figure 2: Bone marrow lesions (WORMS grading) [12]

Subarticular marrow abnormality score. This score is based on the extent of regional marrow involvement by areas of free water signal with ill-defined margins. 
Table 1: Criteria for early OA.

\begin{tabular}{|c|c|c|c|c|c|}
\hline \multicolumn{6}{|c|}{ EARLY OA } \\
\hline \multirow{7}{*}{ 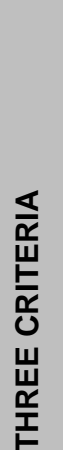 } & 1 & Kne & pain & at least two & des of pain for $>10$ days in the last year \\
\hline & 2 & $\begin{array}{l}\text { Sta } \\
\text { rad }\end{array}$ & $\begin{array}{l}\text { dard } \\
\text { graphs }\end{array}$ & Kellgren-Lav & ce grade 0 or I or II (osteophytes only) \\
\hline & \multirow{5}{*}{3} & \multirow{5}{*}{ 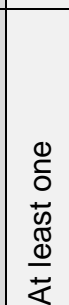 } & arthroscopy & $\begin{array}{l}\text { ICRS grade } \\
\text { or } \\
\text { grade II-IV in }\end{array}$ & $\begin{array}{l}\text { in at least two compartments } \\
\text { compartment with surrounding softening and swelling }\end{array}$ \\
\hline & & & \multirow{4}{*}{ MRI } & \multirow{4}{*}{ At least two } & Cartilage morphology WORMS 3 to 6 \\
\hline & & & & & Cartilage BLOKS grade 2 and 3 \\
\hline & & & & & Meniscus BLOKS grade 3 and 4 \\
\hline & & & & & BMLs WORMS 2 and 3 \\
\hline
\end{tabular}

Table 2: Kellgren and Lawrence radiographic OA classification.

\begin{tabular}{|l|l|}
\hline Grade 0 & No changes \\
\hline Grade 1 & Doubtful narrowing of the joint space and possible osteophytic lipping \\
\hline Grade 2 & Definite osteophytes and possible narrowing of the joint space \\
\hline Grade 3 & $\begin{array}{l}\text { Moderate multiple osteophytes, definite narrowing of the joint space, and some } \\
\text { sclerosis, and possible deformity of the bone ends }\end{array}$ \\
\hline Grade 4 & $\begin{array}{l}\text { Large osteophytes, marked narrowing of the joint space, severe sclerosis, and } \\
\text { definite deformity of the bone ends }\end{array}$ \\
\hline
\end{tabular}

Table 3: Delineation of grading for Cartilage Score 1 [5]

\begin{tabular}{|l|l|}
\hline $\begin{array}{l}\text { Size of any cartilage loss (including partial and full } \\
\text { thickness loss) as a \% of surface area as related to } \\
\text { the size of each individual region }\end{array}$ & thickness cartilage loss of the region \\
\hline $0:$ none & $0:$ none \\
\hline $1:<10 \%$ of region of cartilage surface area & $1:<10 \%$ of region of cartilage surface area \\
\hline $2: 10-75 \%$ of region of cartilage surface area & $2: 10-75 \%$ of region of cartilage surface area \\
\hline $3:>75 \%$ of region of cartilage surface area & $3:>75 \%$ of region of cartilage surface area \\
\hline
\end{tabular}

Table 4: Meniscal tear score (medial meniscus) [10]

\section{Grade 0 : intact}

Grade 1 : minor radial tear or parrot-beak tear

Grade 2 : non-displaced tear or prior surgical repair

Grade 3 : displaced tear or partial resection

Grade 4 : complete maceration/destruction/resection 

Research Coordination Office

Huis Bethlehem

Schapenstraat 34

B-3000 Leuven

Tel.: +3216324065

Fax: +3216324198

onderzoek@kuleuven.be

www.kuleuven.be 